\title{
ARTICLE Methylation of the FKBP5 gene in association with FKBP5 genotypes, childhood maltreatment and depression
}

\author{
Johanna Klinger-König (iD) , Johannes Hertel ${ }^{1,2}$, Sandra Van der Auwera ${ }^{1,3}$, Stefan Frenzel ${ }^{1}$, Liliane Pfeiffer ${ }^{4,5}$, Melanie Waldenberger ${ }^{4,5,6}$, \\ Janine Golchert ${ }^{7}$, Alexander Teumer $\mathbb{1}^{8}$, Matthias Nauck ${ }^{9,10}$, Georg Homuth ${ }^{7}$, Henry Völzke ${ }^{8,10}$ and Hans J. Grabe $\mathbb{D}^{1,3}$
}

DNA methylation of the FKBP5 gene is assumed to alter FKBP5 expression and hence the synthesis of the FK506 binding protein 51 , a central element of a genomic negative feedback loop for glucocorticoid receptor signaling. The present study aimed to replicate and extend previously reported influences of FKBP5 genotypes, childhood maltreatment and depression on methylation levels of five CpG sites in intron 7 of the FKBP5 gene in a large population-based sample. Besides the single nucleotide polymorphism (SNP) rs1360780, associations of the FKBP5 methylation with 22 other, unlinked FKBP5 SNPs as well as associations between FKBP5 methylation levels and transcription levels were investigated. Using whole-blood methylation of 3965 subjects of the Study of Health in Pomerania (SHIP) reduced methylation levels in $\pi$ allele carriers of rs1360780 $(\mathrm{OR}=0.975, p=.005)$ and currently depressed subjects (OR $=0.995, p=0.005)$ were found. Further, an impact of two yet undescribed SNPs (rs6910300, rs7771727) on methylation levels was observed. However, main and interactive effects for childhood maltreatment and lifetime major depressive disorder observed in previous studies could not be replicated. Finally, FKBP5 methylation levels were not related to FKBP5 transcription levels in whole blood. Thus, the present study verified the associations of FKBP5 genotypes and state depression on the FKBP5 methylation levels of five CpG sites in intron 7. However, FKBP5 methylation of these five CpG sites could not be validated as a valuable clinical biomarker for biological long-term effects of childhood maltreatment or lifetime depression.

Neuropsychopharmacology (2019) 44:930-938; https://doi.org/10.1038/s41386-019-0319-6

\section{INTRODUCTION}

To date, the assessment of the psychobiological impact of childhood maltreatment (CM) is limited to interviews and questionnaires [1]. A reliable biomarker reflecting the biological long-term impact of CM would be of high clinical interest. Based on previous studies, altered methylation levels of the FKBP5 gene could putatively serve as such a biomarker. The FKBP5 gene expression and hence the synthesis of the coded FK506 binding protein 51 (FKBP5) is activated by glucocorticoid receptor (GR) signaling [2-5]. The cytoplasmatic FKBP5 protein acts as a cochaperone, binds to the GR and inhibits its translocation into the nucleus $[2,4]$. Hence, FKBP5 is a central element of a genomic negative feedback loop by regulating the GR activity and cell sensitivity towards cortisol stimulus [3,6-8]. According to Klengel et al. [5], the T allele of the single nucleotide polymorphism (SNP) rs1360780 alters the chromatin interaction between the transcription site and long-range enhancers in the FKBP5 gene. Hence, the $T$ allele has been associated with an increased synthesis of FKBP5, a reduced GR sensitivity and thus higher glucocorticoid resistance $[2,5,9,10]$.
Combined with $\mathrm{CM}$, the rs1360780 genotype was identified as an important factor in gene-environment-interactions predicting stress-related disorders like major depressive disorder (MDD) $[6,11,12]$ or posttraumatic stress disorder (PTSD) [7, 13]. Moreover, combined with stressful life events, associations of rs 1360780 with gray matter volume loss, particularly in the hippocampus were reported [5, 14-16]. Although interactive effects between rs 1360780 and $C M$ in relation to mental disorders are well documented $[6,7,11]$, the putative underlying epigenetic mechanisms are still elusive.

According to Klengel et al. [5], the enhanced risk of stressrelated disorders in T allele carriers is mediated by reduced DNA methylation in glucocorticoid response elements of the FKBP5 gene after CM exposure. DNA methylation is an essential part of gene regulation and associated with homoeostatic functioning like genomic imprinting, silencing of repetitive DNA elements and proper expression of genetic information [17-21]. Interactive effects between the rs1360780 genotype and stressful life events were reported to reduce the methylation levels [5, 9, 10, 21]. In addition, reduced methylation levels in intron 7 of the FKBP5 gene

\footnotetext{
${ }^{1}$ Department of Psychiatry and Psychotherapy, University Medicine Greifswald, Ellernholzstraße 1-2, Greifswald 17489, Germany; ${ }^{2}$ Luxembourg Centre for Systems Biomedicine,

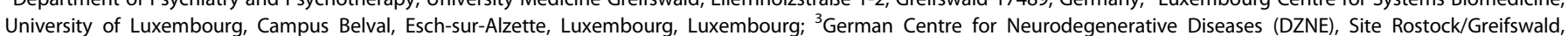

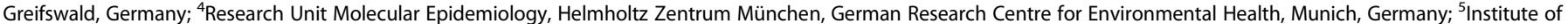

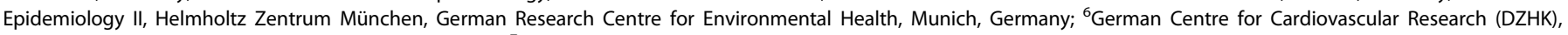

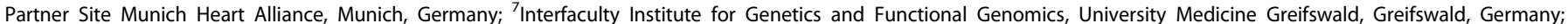

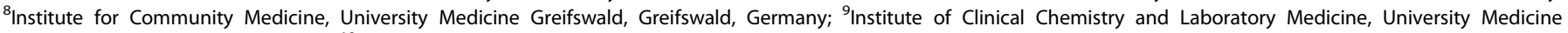
Greifswald, Greifswald, Germany and ${ }^{10}$ German Centre for Cardiovascular Research (DZHK), Partner Site Greifswald, Greifswald, Germany

Correspondence: Sandra Van der Auwera (auweras@uni-greifswald.de)

These authors contributed equally: Johanna Klinger-König, Johannes Hertel
}

Received: 3 September 2018 Revised: 10 January 2019 Accepted: 13 January 2019

Published online: 23 January 2019 
were associated with an enhanced cortisol secretion after stress exposure such as caused by Holocaust exposure [10], preterm birth [21], CM [5, 9] or even laboratory stress tests [22], and a blunted FKBP5 expression [5, 9].

However, most studies focusing on the relations of methylation levels, traumatic experiences and mental health symptoms were based on patient samples and/or small sample sizes. As we have available different layers of biological data (SNP information, gene-expression and methylation of FKBP5) as well as detailed information of phenotypes (lifetime MDD, current depressive symptoms, CM exposure), we aim to shed light on the complex mechanisms underlying the associations between the different measurements. In addition, our data could give certainty whether methylation levels were stronger associated with state (lifetime MDD) or trait depression (current depressive symptoms). Because of the lack of longitudinal data, we test for associations assuming different biological models with either methylation or depression as end point. In a first step, we investigated the direct and interactive associations of CM, rs1360780 and depression on FKBP5 methylation levels of five previously reported CpG sites in intron 7 [5] and the mean methylation level across these sites in two large general population-based samples using data from the Study of Health in Pomerania (SHIP). We hypothesised the SNP rs 1360780 to have an impact on the methylation levels in intron 7 with even stronger effects in subjects with $\mathrm{CM}$ and depression. Exploratory, we analysed the same associations including direct and interactive effects for 22 other independent FKBP5 SNPs. In a second step, we tested for interactive effects of SNPs, CM and methylation on the end point state/trait depression. At last, we exploratory analysed the direct and interactive effects of SNPs, $\mathrm{CM}$, methylation and depression on the FKBP5 transcription levels in a subsample.

\section{MATERIALS AND METHODS}

Study population

We analysed data from the SHIP, a two-stage cluster sample of adult German residents of north-eastern Germany randomly drawn from local registries [23]. The baseline sample (SHIP-0: 1997-2001) comprised of 4308 Caucasians, ages 20-79 years. Three follow-up assessments have been carried out (SHIP-1: 2002-2006, SHIP-2: 2008-2012, SHIP-3: 2015-2017). In parallel to SHIP-2 $(N=2333)$, the study Life Events and Gene-EnvironmentInteraction in Depression (SHIP-LEGENDE: 2007-2010) was conducted. Out of the baseline sample (SHIP-0), 2400 subjects participated in SHIP-LEGENDE which comprised detailed assessments of life events and mental disorders. For 1841 subjects of SHIP-2 (Table 2) and SHIP-LEGENDE full data for the present analyses were available.

A second, independent cohort, SHIP-TREND-0 $(N=4420)$, was drawn from local registries of the same area as SHIP-0 from 2008 till 2011. Note that the samples of SHIP-0 and SHIP-TREND-0 are without overlap and sampled independently of each other. For 2124 subjects of SHIP-TREND-0 (Table 2), methylation status was analysed. Genotyping of the SNP rs1360780 was done for all of these subjects. For a random sample of 1000 subjects (herein called "SHIP-TREND-OMICS", excluding diabetics) comprehensive multi-omics characterisation and whole-genome chip genotyping were performed. Hence, genotypes of the whole FKBP5 gene were available for 959 subjects and FKBP5 transcription levels were available for 938 subjects. Analysis of OMICS data has been done with whole blood collected at one time point.

SHIP and all analyses conducted in the present study were performed in accordance with the Declaration of Helsinki, including written informed consent of all subjects. The survey and methods of the SHIP studies were approved by the institutional review board of the University Greifswald.
SNP-typing and imputation

The SHIP-2 sample was genotyped using the Affymetrix Human SNP Array 6.0 (Affymetrix, Santa Clara, CA, USA). Sample processing and array hybridisation were done in accordance with the manufacturer's standard recommendations. For further details and imputation procedure, see Völzke et al. [23] and the supplement. Genotyping of the SHIP-TREND-OMICS subsample was performed using the Illumina HumanOmni 2.5-Quad (Illumina, San Diego, CA, USA). DNA from whole blood was prepared using the Gentra Puregene Blood Kit (Qiagen, Hilden, Germany) according to the manufacturer's protocol. Subsequent sample processing and array hybridization were performed as described by the manufacturer at the Helmholtz Center Munich. Again, for further details and imputation procedure, see Völzke et al. [23] and the supplement.

To select further FKBP5 SNPs besides rs1360780, all SNPs of the FKBP5 gene within $10 \mathrm{kB}$ were pruned with $R^{2}<0.25$ and were checked for their minor allele frequency (MAF) and imputation quality. For all SNPs, an imputation quality of at least $80 \%$ was required. Further, for main effect analyses, SNPs were selected if the MAF was higher than 5\%. Hence, 23 SNPs (including rs1360780) were selected for analyses (Table 1). However, as larger group sizes are needed to test interaction effects, only a subsample of 8 SNPs (rs1360780, rs6910300, rs3798346, rs4713904, rs9368882, rs55987213, rs2817032, and rs2817035) was used in the interaction analyses determined by a MAF higher than $20 \%$. The observed by expected variance ratio of the SNPs used in the present study varied between 0.86 and 0.997 for SHIP0 and between .88 and .999 for SHIP-TREND-0 which indicates a high imputation quality.

Whole-blood DNA methylation data

The methylation state of five CpG sites in the intron 7 of the FKBP5 gene was measured by MALDI-TOF mass spectrometry using EpiTYPER by MassARRAY (Sequenom, San Diego, CA), using whole blood as previously described [24]. For further details, see supplement.

Whole-blood transcriptome levels

Sample collection and whole-blood RNA preparation were described in detail elsewhere [25]. For a brief description, see the supplement. For the present study, we extracted the transcript level data of the gene FKBP5 (only one transcript available ID: ILMN_1778444). We regressed the quantile normalised and log2 transformed transcript level quantification on technical covariates (time of blood sampling, RNA integrity number as a measure of RNA quality, RNA amplification batch), blood cell counts, haematocrit, mean platelet volume, age, and gender and then derived the residual variables. This residual variable was used for association analyses.

\section{Interview and psychometric data}

Sociodemographic information and medical history were assessed by a computer-assisted face-to-face interview. In both cohorts (SHIP-2 and SHIP-TREND-0), CM was assessed using the Childhood Trauma Questionnaire (CTQ) which indicates more severe CM by higher scores in the CTQ sum score and five subscales (emotional/ physical/sexual abuse, emotional/physical neglect), respectively [26]. The present study focused on the CTQ sum score, only including the CTQ subscales as sensitivity analyses if the sum score revealed significant results. Lifetime MDD was assessed using the Munich-Composite International Diagnostic Interview (M-CIDI) in SHIP-2, a highly reliable, standardised and structured instrument assessing psychiatric disorders according to the DSMIV criteria [27, 28]. Moreover, the severity of depressive symptoms was assessed using the Beck-Depression-Inventory-II (BDI-II) [29]. In SHIP-TREND-0 the Patient Health Questionnaire (PHQ-9) was used to assess both lifetime MDD (dichotomous evaluation) and 
Table 1. Minor allele frequencies of the FKBP5 SNPs for SHIP-2 and SHIP-TREND-OMICS

\begin{tabular}{|c|c|c|c|c|c|c|c|}
\hline & Common allele & Minor allele & \multicolumn{3}{|c|}{ Minor allele frequency (\%) } & \multicolumn{2}{|c|}{ Imputation quality } \\
\hline rs1360780a & C & $\mathrm{T}$ & 30.45 & 30.04 & 31.37 & 1.00 & 1.00 \\
\hline $\mathrm{rs} 2817035^{\mathrm{a}}$ & G & A & 30.27 & 30.77 & 29.30 & 0.95 & 0.99 \\
\hline $\operatorname{rs} 2817032^{\mathrm{a}}$ & $\mathrm{T}$ & $C$ & 30.21 & 30.74 & 29.20 & 1.00 & 1.00 \\
\hline rs3798346 ${ }^{\mathrm{a}}$ & $A$ & G & 23.25 & 23.66 & 22.47 & 0.97 & 0.99 \\
\hline rs55987213 ${ }^{a}$ & $\mathrm{C}$ & $\mathrm{T}$ & 20.64 & 20.42 & 21.06 & 0.99 & 0.99 \\
\hline rs12197246 & G & A & 19.43 & 19.55 & 16.71 & 0.98 & 0.99 \\
\hline rs55694295 & C & $\mathrm{T}$ & 18.93 & 18.82 & 19.13 & 0.99 & 0.99 \\
\hline rs12200498 & G & $A$ & 18.57 & 18.69 & 18.35 & 1.00 & 1.00 \\
\hline rs9470079 & G & $A$ & 16.71 & 17.00 & 16.16 & 0.86 & 0.99 \\
\hline rs7740395 & $\mathrm{T}$ & C & 16.59 & 16.08 & 17.57 & 0.96 & 0.99 \\
\hline rs4713899 & G & A & 15.70 & 15.51 & 16.06 & 1.00 & 1.00 \\
\hline rs7771727 & G & $A$ & 13.75 & 13.66 & 13.92 & 0.99 & 1.00 \\
\hline rs72921231 & $\mathrm{T}$ & C & 10.30 & 10.67 & 9.59 & 1.00 & 1.00 \\
\hline rs72913423 & A & G & 10.09 & 10.35 & 9.59 & 0.99 & 1.00 \\
\hline rs72913412 & G & A & 10.09 & 10.35 & 9.59 & 0.99 & 0.99 \\
\hline rs2294807 & $C$ & $\mathrm{~T}$ & 10.05 & 10.32 & 9.54 & 1.00 & 1.00 \\
\hline rs74709645 & $C$ & $\mathrm{~T}$ & 5.79 & 5.95 & 5.47 & 0.79 & 0.97 \\
\hline
\end{tabular}

current depressive symptoms (metric evaluation). To optimise the comparability between the two cohorts, the metric PHQ-9 score was transformed into a BDI-II score. The methodical background of this procedure was described in detail by Wahl et al. [30].

\section{Statistical analyses}

For descriptive statistics, metric variables were expressed in means (M) and standard deviations (SD); categorical variables were expressed in proportions. All regression analyses were focused on the combined sample to maximise statistical power.

All statistical analyses were performed using STATA/SE 14.2 (STATA corp, College Station, Texas, USA). A nominal significance level of $p$-value $(p)=0.05$ was used. A Bonferroni-correction was used to correct for multiple testing. The corrected $p$-values for each class of analyses are reported below and in the results section. Effects were considered as valid if (1) the p-value of the combined analysis (SHIP-2 + SHIP-TREND) reached the corrected significance level and (2) the effects in both cohorts were in the same direction, as a meaningful biological effect was assumed to cause the same statistical effect direction independently of the sample. Genetic association results were treated as meaningful when the group of homozygote risk allele carriers for a SNP were at least $N=10$ subjects. All $\mathrm{p}$-values reported are two-tailed. Analyses including the CTQ subscales instead of the sum score were conducted only if there was a significant result using the CTQ sum score.

Covariate list. All basic analyses were adjusted for age, gender and cohort. However, as whole-blood DNA methylation levels were used, an extended covariate list was used for sensitivity analyses, too (see Table S1). Therewith, potential influences of smoking, waist circumference, white and red blood cell counts, platelet counts and mean platelet volume, fibrinogen, glycated haemoglobin, cystatin C, triglycerides and time of blood sampling on the methylation levels of the five CpG sites were tested. Data for the different cell types of white blood cells (monocytes/ lymphocytes/eosinophils/basophils/neutrophils) only were available for the SHIP-TREND-0 sample.

Hypotheses 1. We tested main effects of CM (CTQ sum score), depression (lifetime MDD, BDI-II score) and the SNP rs1360780 on the methylation level of five distinct CpG sites of intron 7 of the FKBP5 gene and the mean methylation level across these five sites. In addition, the two-way interactions $(\mathrm{CM} \times$ depression, $\mathrm{CM} \times \mathrm{SNP}$, depression $\times$ SNP) and the three-way-interactions $(C M \times$ depression $\times$ SNP) on the methylation levels were tested. As methylation data are fractional data (between 0 and 1), a semiparametric regression methodology developed to deal with fractional data was used [31, 32]. This regression methodology does not rely on distributional assumptions and only needs the specification of the functional form of the predictive mean which was chosen to be logistic in our case. The variance estimators are inherently robust against heteroscedasticity and the regression coefficients are easily interpretable as they can be expressed in odds ratios. All hypotheses were tested on five CPG sites and the mean methylation level across these sites. Hence, a Bonferronicorrected significance level of $p=0.008$ (six tests) was used to adjust for multiple testing.

This set of analyses was repeated to test for altered methylation levels associated with 22 other FKBP5 SNPs (Table 1). Direct effects on the five CpG sites and mean methylation levels as well as 
two-way interactions (SNPs $\times$ CM, SNPs $\times$ depression) and threeway interactions (SNPs $\times \mathrm{CM} \times$ depression) were tested. Note that, due to a narrower MAF ( $>20 \%)$ required for the interaction effects, only seven SNPs (rs6910300, rs3798346, rs4713904, rs9368882, rs55987213, rs2817032 and rs2817035; Table 1) entered the interaction analyses. Again, the semiparametric regression methodology described above was used. A Bonferroni-corrected significance level of $p=3.79 \mathrm{e}-04$ (132 tests) was used to adjust for multiple testing because analyses were conducted on six methylation outcomes (five $\mathrm{CpG}$ sites and the mean methylation level) investigating the main effects of 22 other FKBP5 SNPs.

Hypotheses 2: Effects of methylation in predicting depression. Using lifetime MDD as an outcome, logistic regression analyses were calculated. For the severity of current depressive symptoms, the BDI-II score was used as an outcome in bootstrapped linear regression analyses. The additive effect of integrating methylation into the two-way-interactions (SNPs $\times$ methylation, $\mathrm{CM} \times$ methylation), as well as the three-way-interactions (SNPs $\times \mathrm{CM} \times$ methylation), were checked, respectively, using a likelihood ratio test. All 8 SNPs appropriate to test interaction effects (rs1360780, rs6910300, rs3798346, rs4713904, rs9368882, rs55987213, rs2817032, rs2817035; MAF > 20\%) were used linearly. As two depression outcomes (lifetime MDD and the BDI-II score) were used and six methylation variables (five CPG sites and the mean methylation level), as well as eight FKBP5 SNPs, were included as predictors, a Bonferroni-corrected significance level of $p=5.21 \mathrm{e}-04$ (96 tests) was used.

Hypotheses 3: Main and additional effects of methylation on the FKBP5 whole-blood transcription levels. Linear regression analyses were calculated. Main effects of methylation as well as the additive effect of integrating methylation into the two-wayinteractions (SNPs $\times$ methylation, $\mathrm{CM} \times$ methylation, depression $\times$ methylation), the three-way-interactions (SNPs $\times \mathrm{CM} \times$ methylation, SNPs $\times$ depression $\times$ methylation, $C M \times$ depression $\times$ methylation) and the four-way interactions (SNPs $\times C M \times$ depression $\times$ methylation) were analysed. Again, the eight SNPs appropriate to test interaction effects were used. Further, note that the FKBP5 transcription levels were available only for subjects of SHIPTREND-OMICS. All analyses were adjusted for age and gender. As six methylation variables (five $\mathrm{CpG}$ sites and the mean methylation level), eight FKBP5 SNPs and two depression variables (lifetime MDD and the BDI-II score) were used, a Bonferroni-corrected significance level of $p=5.21 \mathrm{e}-04$ (96 tests) was used.

Sensitivity analyses. All analyses including FKBP5 SNPs were calculated twice, using the SNPs as linear and as categorical variables, respectively. Categorical analyses are presented in the supplement. Additionally, all analyses were performed also adjusting for the extended covariate list in the regression model.

\section{RESULTS}

Descriptive statistics of the study samples are presented in Table 2. As SHIP-2 is the 10-year-follow-up of SHIP-0 and SHIP-TREND-0 is an independent new baseline cohort, the SHIP-2 cohort was older (Table 2) and the samples differed regarding somatic and blood parameters (supplementary table S1). Methylation levels differed between SHIP-2 and SHIP-TREND-0 on four of the five CpG sites but did not differ significantly regarding the mean methylation of intron 7. Descriptive statistics for SHIP-TREND-OMICS including transcriptome data as well as comparisons between SHIP-TRENDOMICS and SHIP-2 are presented in tables S2a-c.

Extended covariate list

Extensive associations between methylation levels and the tested covariates were found: For SHIP-2, the mean methylation level was associated with all covariates, except of smoking, waist circumference and cystatin C. In SHIP-TREND-0, the mean methylation level was associated with age, gender, smoking, waist circumference, serum triglycerides, and red and white blood cell count. The influences of the covariates on the five CpG sites varied widely between the cohorts (SHIP-2 and SHIP-TREND), summarised in Tables S2a-S2c. The different cell types of white blood cells showed no significant associations with the methylation levels (Table S3). Due to these extensive associations, sensitivity analyses using the extended covariate list were conducted for all analyses reported below.

Hypotheses 1

Results are presented in Table S4. A Bonferroni-corrected significance level of $p=0.008$ (six methylation variables) was used. The SNP rs1360780 was associated with a reduced methylation on CpG site 2 (OR $=0.975,95 \% \mathrm{Cl}$ : $0.957-0.992$, $p=0.005$ ). Additionally, the BDI-II score was associated with a reduced methylation ( $\mathrm{OR}=0.995,95 \% \mathrm{Cl}$ : 0.992-0.999, $p=$ 0.005 ) on CpG site 4 as well as a two-way-interaction between the BDI-II score and rs 1360780 (OR $=1.007,95 \% \mathrm{Cl}: 1.002-1.012$, $p=0.006$, Fig. 1). The effect sizes of these associations were similar in both cohorts (Table S4). All effects remained stable adjusting the model for the extended covariate list. There were neither direct effects of $\mathrm{CM}$ nor interactive effects of $\mathrm{CM}$ and rs1360780 observable.

Regarding the other 22 independent FKBP5 SNPs a Bonferronicorrected significance level of $p=3.79 \mathrm{e}-04$ (six methylation variables $\times 22$ FKBP5 SNPs) was used. Using the linear SNP variables, none of the tested models did reach the corrected $p$ value. However, using the categorical SNP variables, main effects of three SNPs (rs6910300, rs7771727, rs74709645) were observed (supplementary results), presented in Fig. 2. As the SNP rs74709645 had a very low MAF of $5.79 \%$ with only seven homozygote risk allele carriers in the combined sample, we did not present this finding as a reliable result. No significant interactions were observed. All effects remained stable adjusting the model for the extended covariate list.

Hypotheses 2: Effects of methylation in predicting depression Analyses were calculated to investigate whether the integration of the methylation variables to the interaction terms (methylation $\times$ FKBP5 SNPs, methylation $\times C M$, methylation $\times F K B P 5$ SNPs $\times$ CM) improved the prediction of depression measured by two outcome variables (lifetime MDD and the BDI-II score). A Bonferroni-corrected significance level of $p=5.21 \mathrm{e}-04$ (2 depression outcomes $\times 6$ methylation variables $\times 8$ FKBP5 SNPs) was used. There were no significant effects using lifetime MDD as the outcome variable, neither using the linear nor using the categorical SNP variables. Using the BDI-II score, an improved prediction was observed by including the methylation on CpG site 4 in the two-way-interaction with rs1360780 $\left(\mathrm{chi}^{2}=\right.$ 15.32, $p=4.71 \mathrm{e}-04$, increase in $\left.R^{2}: 0.37 \%\right)$, replicating the interaction effect of rs1360780 and depressive symptoms on CpG site 4 (described above), as well as by including the methylation of $\mathrm{CpG}$ site 4 in the three-way-interaction with rs 1360780 and the CTQ sum score $\left(\mathrm{chi}^{2}=27.72, p=1.42 \mathrm{e}-05\right.$, increase in $\left.R^{2}: 0.69 \%\right)$. However, the improvements of prediction by including two-way and three-way interaction terms, although significant, were very small. Unexpectedly, there was an inverse relation of $\mathrm{BDI}-\mathrm{II}$ and methylation only for CC-homozygote carriers reporting high levels of CM (figure S1). In sensitivity analyses, CTQ subscales were included into the three-wayinteraction. Only for emotional abuse, a significant effect was observed in the combined sample $\left(b=7.23\right.$, chi $^{2}=28.88, p=$ 8.27e-06). However, the interaction effect was positive in SHIP-2 $(b=19.60)$ but inverse in SHIP-TREND-0 $(b=-0.85)$. All results were validated with the extended covariate list. 
Table 2. Descriptive statistics of SHIP-2 and SHIP-TREND

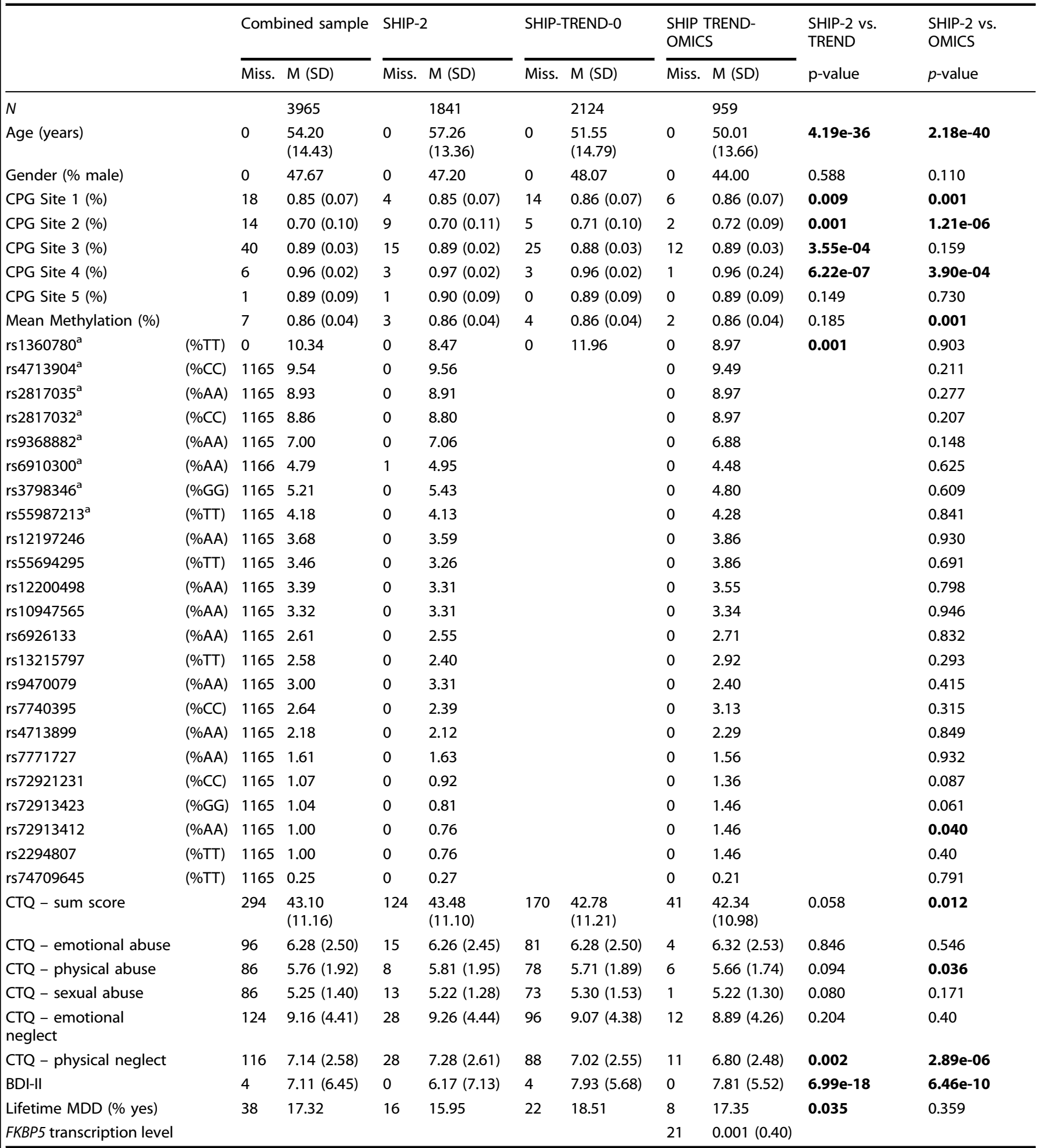

CTQ Childhood Trauma Questionnaire, BDI-II Beck Depression Inventory-II, MDD major depressive disorder

${ }^{\text {a }}$ SNPs used in the interaction effect analyses

Bold values in table 2 are nominal significant associations

Hypotheses 3: Main and additional effects of methylation in predicting the FKBP5 transcription levels

Main effects of the methylation levels and additive effects of methylation levels in interaction models with 8 FKBP5 SNPs and depression (lifetime MDD and BDI-II) in predicting the FKBP5 whole-blood transcription levels were analysed. A Bonferronicorrected significance level of $p=5.21 \mathrm{e}-04$ (6 methylation variables $\times 8$ FKBP5 SNPs $\times 2$ depression variables) was used. No significant main or additive effects were observed, neither using the linear nor using the categorical SNP variables. 
A

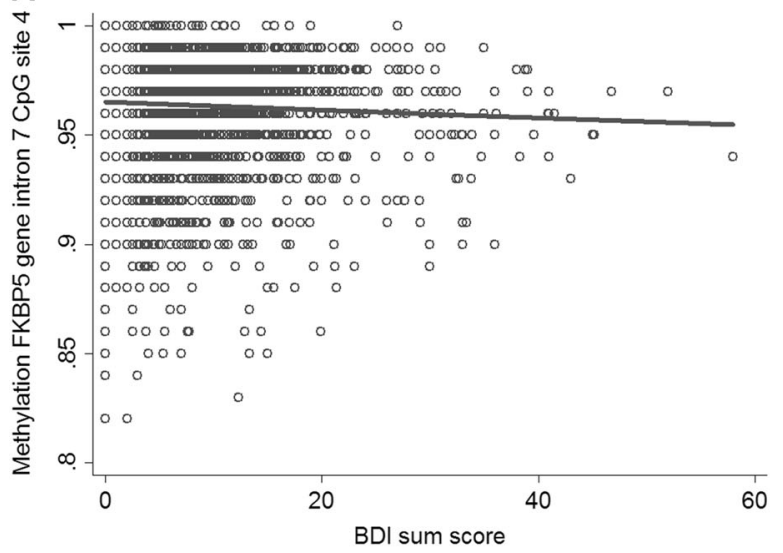

B

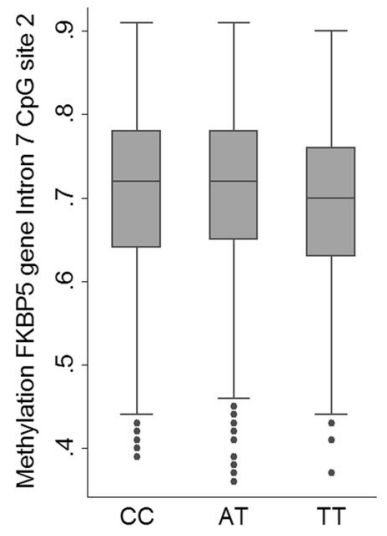

C

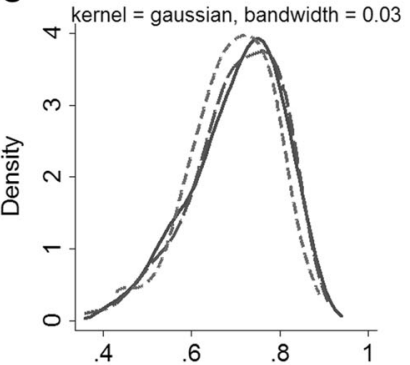

Methylation FKBP5 gene Intron 7 CpG site 2

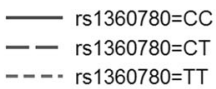

D

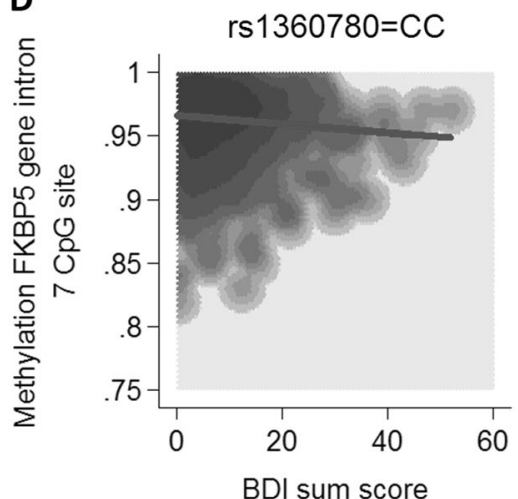

rs $1360780=\mathrm{CT}$

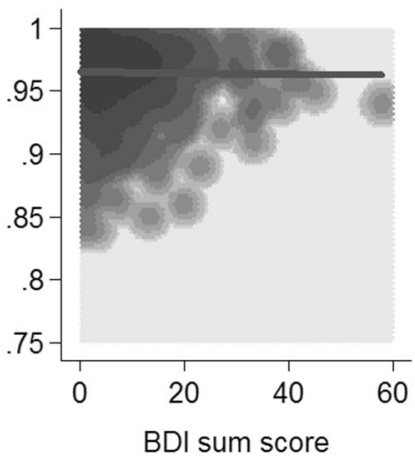

rs $1360780=$ TT

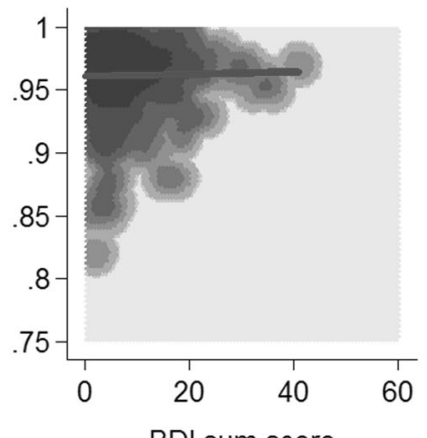

Fig. 1 Main and interaction effects of current depressive symptoms (BDI-II) as well as the FKBP5 SNP rs1360780 on the FKBP5 DNA methylation level. a Higher scores of the BDI-II score are associated with reduced methylation on CpG site $4(\mathrm{OR}=0.995, p=0.005)$. $\mathbf{b}$ Box plots of methylation on CpG site 2 over rs1360780. The minor T allele of rs 1360780 is associated with reduced methylation on CpG site 2 (OR=0.975, $p=0.005)$. c Kernel density plots of methylation on CpG site 2 over rs 1360780 in dependency of rs 1360780 using Gaussian kernels. d 2D kernel density plots of the bivariate BDI-methylation distribution on CpG site 4 using Gaussian kernels stratified for rs 1360780 genotypes. For homozygote C allele carriers of rs1360780, a higher BDI-II score was associated with reduced methylation on CpG site 4 (OR=0.991, $p=1.36 \mathrm{e}-04$ ), but not for subjects carrying the minor $\mathrm{T}$ allele $(\mathrm{OR}=1.004, p=0.428)$

\section{DISCUSSION}

The aim of the present study was to rigorously test the validity and generalisability of previous findings connecting FKBP5 SNP genotypes and CM with FKBP5 gene hypomethylation leading to increased FKBP5 transcription levels and to the burden of depressive symptoms.

Klengel et al. [5] published a comprehensive study investigating the associations of the rs1360780 genotype, FKBP5 methylation levels, and psychiatric symptoms after the experience of $C M$. The authors reported reduced methylation levels in intron 7 for carriers of the T risk allele, for subjects reporting $\mathrm{CM}$ and even stronger hypomethylation for subjects being both carriers of the T risk allele and maltreated in childhood $[5,10,16]$. In extension, other studies reported higher methylation levels for MDD patients with the effect being even more attenuated in $\Pi$ carriers $[9,16,33]$. Nevertheless, few putative covariates of gene methylation were included in these studies. Our data revealed extensive associations between FKBP5 methylation levels in intron 7 and somatic as well as blood parameters (see Table S2).

Even though our data partly replicated the reduced methylation levels in T allele carriers as well as inverse associations between methylation levels and depressive symptoms, we could neither replicate relations of methylation and CM nor between methylation and lifetime MDD. No main effect or interaction effects with CM did reach significance level. Even though CTQ subscales did reveal some significant results (results not presented), findings were inconsistent between cohorts and regarding the $\mathrm{CpG}$ sites as well as the effect directions. In general, in comparison to earlier studies $[5,10]$, we report higher general methylation levels and descriptively smaller differences. All effects identified were rather small in terms of effect sizes. Furthermore, we note that previous studies used statistical methods from the class of the general linear model (e.g. ANOVA). However, it is well known that in fractional data the variance is a function of the expectation [34], invalidating theoretically the assumptions inherent to the general linear model and questioning the statistical robustness of the effect sizes reported earlier. In contrast, we use here a semiparametric methodology explicitly designed to analyse fractional data [32], which expresses its effect sizes in odds ratios. As such, our results have to be interpreted as changes in the odds that a certain CpG-site is methylated, rather than differences in mean methylation levels.

Predicting methylation levels in adulthood altered through CM relies on long-lasting effects [35-39].

Besides studies reinforcing associations between altered FKBP5 methylation levels and CM $[5,9]$, others have focused on the fast adaptability of methylation. Hence, Piyasena et al. [21] reported the lower FKBP5 methylation levels of preterm compared to term infants to be not observable anymore at one year of age indicating an adaptation of methylation within the first year of life. Even faster alterations of methylation were shown in laboratory tests. In rodents, Lee et al. [40] observed reduced methylation levels in neuronal and blood cells after 4 weeks of providing water containing corticosterone. Winkler et al. [22] 
A

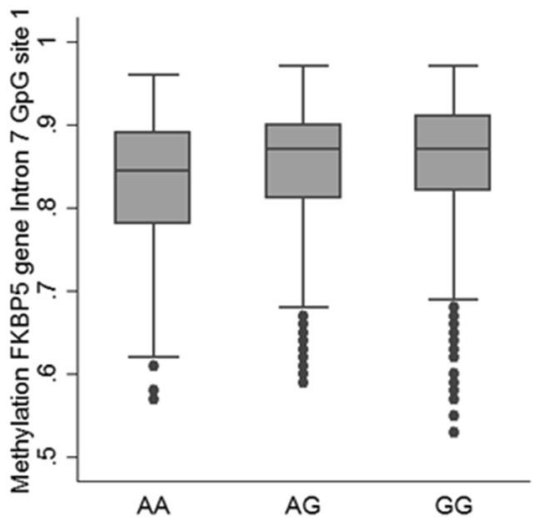

C

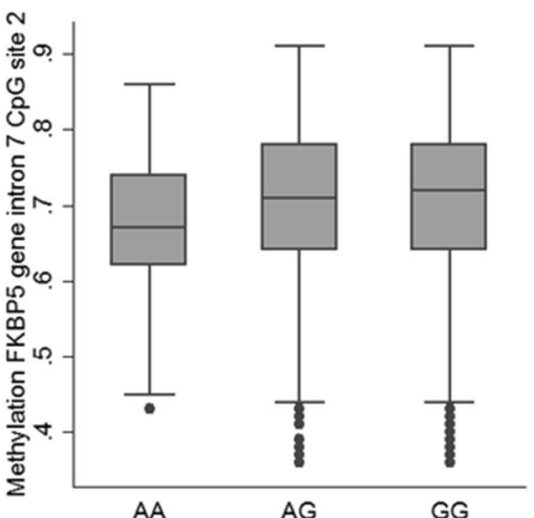

B

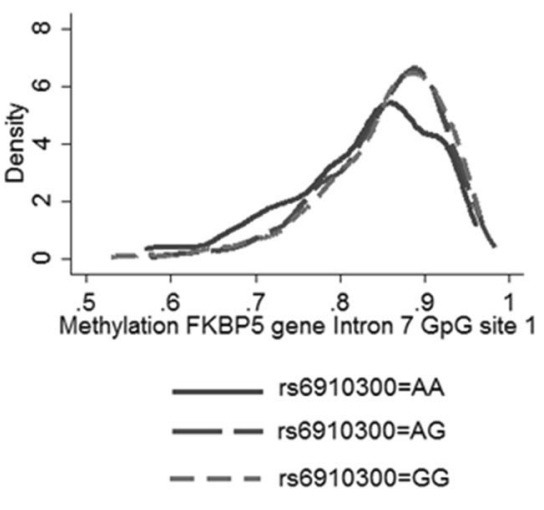

D

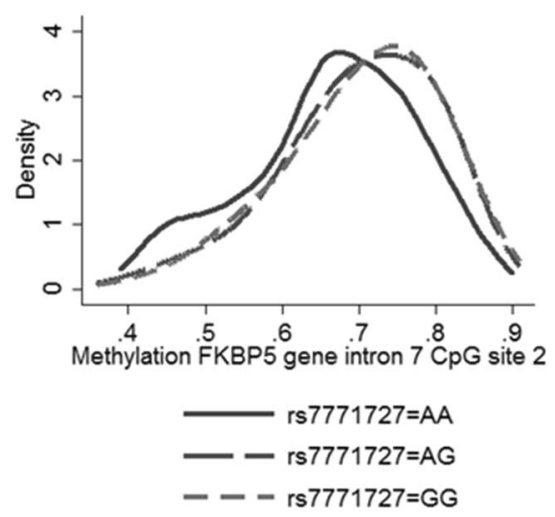

Fig. 2 Main Effects of the FKBP5 SNPs rs6910300 and rs7771727 on the FKBP5 DNA methylation level. a Box plots of methylation on CpG site 1 over rs6910300. The minor A allele of rs6910300 was associated with reduced methylation on CpG site $1\left(\mathrm{chi}^{2}=21.38, p=2.28 \mathrm{e}-05\right)$. b Kernel density plots of methylation on CpG site 1 over rs6910300 in dependency of rs6910300 using Gaussian kernels. c Box plots of methylation on CpG site 1 over rs7771727. The minor A allele of rs7771727 was associated with reduced methylation on CpG site 1 (chi² $=17.64, p=1.47$ - 04 ). d Kernel density plots of methylation on CpG site 1 over rs7771727 in dependency of rs7771727 using Gaussian kernels

reported altered methylation levels in human leucocytes $24 \mathrm{~h}$ after an ACTH-stress-test. Thus, DNA methylation might reflect both short-term alterations to adapt to acute environmental changes as well as long-term processes coded by genetic disposition and long-lasting environmental influences like CM or lifetime MDD.

Previous studies used both lifetime MDD and depressive symptoms to operationalise depression $[9,16,33,41]$. Bustamante et al. [41] reported CM to predict the severity of depressive symptoms but did neither observe an association of FKBP5 methylation levels in intron 7 or intron 2 with CM nor with depressive symptoms. Moreover, only lifetime MDD was related to FKBP5 expression [41]. Although Roy et al. [33] reported MDD to be associated with higher FKBP5 methylation levels in the promoter region, effects were mainly carried by suicidal patients who reported more severe depressive symptoms. We investigated whether the associations between methylation levels and depression would be mainly carried by state or trait depression. According to previous studies, we observed an inverse association between the severity of depressive symptoms and FKBP5 methylation levels. This association was influenced by the rs1360780 genotype which is in line with Höhne et al. [9] who reported higher FKBP5 methylation levels in intron 7 in TT carriers with lifetime MDD. Nevertheless, our data did not reveal any main or interactive effects including lifetime MDD.

Besides rs 1360780, in addition to former research, the present study investigated the impact of 22 other, unlinked FKBP5 SNPs on the methylation levels in intron 7. Using the genotypes as categorical variables, main effects of rs6010300 and rs7771727 on methylation levels were observed (supplement) with homozygote carriers of the minor allele of rs6010300 and rs7771727 showing reduced methylation levels. Nevertheless, as there is no previous research including any of these SNPs and as there were no interaction effects with $\mathrm{CM}$ or depression observed in our data, the impact of the main effects of these SNPs stays unclear. The rs1360780 genotype and reduced methylation levels were repeatedly reported to induce higher FKBP5 expression and higher cortisol levels, also associated with higher stress levels $[5,9,22]$. However, neither rs1360780 nor one of the three other SNPs found in the explorative analyses had an interaction effect with methylation on the expression levels in our data. Moreover, even the main effects of methylation on FKBP5 expression were absent. Although previous studies have implicated an inverse relation of methylation levels with FKBP5 expression, it remains uncertain if this relation is observable in baseline levels, as used in the present study, or only appears after acute stress reactions [5, 41-44]. Likewise, Klengel et al. [5] reported no baseline association between methylation and expression levels in blood and neuronal cells but observed an inverse correlation between methylation levels and FKBP5 response to dexamethasone in neuronal cells simulating acute stress [5, 42]. On the other hand, Needham et al. [43] found the same inverse relation even at baseline. In addition, Paquette et al. [44] described this baseline association to be even stronger in TT carriers of rs 1360780 . None of the results could be replicated in our data which is in line with results by Bustamante et al. [41] who did neither observe an effect of CM on the expression nor an association between methylation levels and 
FKBP5 expression. Although all these studies tried to investigate the association between CT, DNA methylation of FKBP5 and depression, they differed in their methylation sites, sample size, ethnicity and sample composition.

\section{Strength and limitations}

Whereas previous studies used relatively small (patient) samples, the present results were based on two large population-based cohorts (SHIP) which were comprised of almost 4000 subjects. Although previous studies focused on a few SNPs, mostly on rs1360780, we used additionally 22 statistically selected FKBP5 SNPs. Moreover, we investigated different associations of FKBP5 methylation with the trait (MDD lifetime) and state depression (BDI-II). Finally, the present study investigated direct effects between FKBP5 methylation and transcription levels in the blood which is thought to be a pathophysiological relevant step. Our population-based sample reported relatively low rates of $\mathrm{CM}$ and less severe depressive symptoms compared to clinical samples. Hence, we might have missed effects due to severe $\mathrm{CM}$ and/or depression. As our data are drawn from a general population sample, the distribution of depressive symptoms and reported CM is relatively low compared to clinical settings (in our clinical sample the average BDI-II score is about four to five times higher and the CTQ score about 1.5 times higher). In a clinical setting you would also get information about comorbid psychiatric disorders which are not available in our population-based sample. Further, as our data was observational and cross-sectional we cannot conclude causality, particularly regarding the associations between methylation levels and depression. Finally, we did focus on the FKBP5 methylation levels in intron 7. However, an FKBP5 gene-wide methylation profile could help to identify other putatively relevant methylation sites. Nevertheless, we used only five CpG sites in intron 7 of the FKBP5 gene in human blood cells. Few studies have been published comparing methylation levels in neuronal and blood cells. Whereas Klengel et al. [5] reported similar methylation levels and similar associations between methylation levels and transcription levels in both cell types, Ewald et al. [45] reported low correlations between methylation levels CpG sites of neuronal and blood cells. Accordingly, Lee et al. [40] reinvestigated the neuronal and blood cells of rodents after 1 week of recovery from being fed with water containing corticosterone and observed a persisting reduction of methylation in both cells. Importantly, after 4 weeks, blood methylation levels returned back to baseline. We focused on direct and interaction effects but did not included mediation analyses. Nevertheless, from a statistical point of view requirements are hardly fulfilled as low to no direct associations were observed between the FKBP5 SNPs and methylation levels and low to no direct associations were observed between depression phenotypes and methylation levels (data not presented). More research is needed to address these questions as well as to replicate and extend our results using other CpG sites or wholegenome methylation data.

\section{Conclusion}

Our large effort to validate and extend previous findings of the FKBP5 methylation resulted in a few significant results only. Results had very small effect sizes; most of the associations were specific to one $\mathrm{CpG}$ site and were inconsistent between the cohorts regarding the effect direction. Our results did not confirm any robust effects of $C M$ on methylation at five sites in intron 7 of the FKBP5 gene nor interaction effects with FKBP5 SNPs. These results make it unlikely that these five methylation sites could serve as a biomarker for individual exposure to $\mathrm{CM}$ and related biological consequences in the general population.

\section{FUNDING AND DISCLOSURE}

SHIP is part of the Community Medicine Research net of the University of Greifswald which is funded by the Federal Ministry of
Education and Research (01ZZ9603, 01ZZ0103, and 01ZZ0403), the Ministry of Cultural Affairs and the Social Ministry of the Federal State of Mecklenburg-West Pomerania. Genome-wide SNP typing in SHIP has been supported by a joint grant from Siemens Healthcare, Erlangen and the Federal Ministry of Education and Research (03ZIK012). JKK, JH, and SV were supported by the German Research Foundation (DFG: GR1912/11-1). In addition, SV was supported by the German Federal Ministry of Education and Research (BMBF) within the framework of the e:Med research and funding concept (Integrament: 01ZX1614E) and SV and JH were supported by the German Research Foundation (CRC-TRR58, projects C02 and Z02 to KD). SF was supported by the EU "Joint Programme Neurodegenerative Disorders" (JPND: 01ED1615). The position of LP was financed through the European Union Seventh Framework Programme under grant agreements $n^{\circ} 261433$ and $n^{\circ} 602736$. MW has a permanent position financed by the Helmholtz Zentrum München - German Research Center for Environmental Health, which is funded by the German Federal Ministry of Education and Research and by the State of Bavaria. M. Waldenberger has received funding from the European Union Seventh Framework Programme under grant agreements no. 261433, no. 313010 , no. 603288 and no. 602736 as well as a grant from the German Research Foundation (WA 4081/1-1). AT was supported by the German Federal Ministry of Education and Research (BMBF) via the DZHK (German Center for Cardiovascular Research: $81 \times 34000104)$. JG and GH received research funding from the German Research Foundation (DFG). HJG has received travel grants and speakers honoraria from Fresenius Medical Care and Janssen Cilag. He has received research funding from the German Research Foundation, the German Federal Ministry of Education and Research (BMBF), the DAMP Foundation, Fresenius Medical Care, the EU "Joint Programme Neurodegenerative Disorders" (JPND: 01ED1615) and the European Social Fund (ESF).

\section{ADDITIONAL INFORMATION}

Supplementary Information accompanies this paper at (https://doi.org/10.1038/ s41386-019-0319-6).

Competing interests: The authors declare no competing interests.

Publisher's note: Springer Nature remains neutral with regard to jurisdictional claims in published maps and institutional affiliations.

\section{REFERENCES}

1. König J, Block A, Becker M, Fenske K, Hertel J, Van der Auwera S, et al. Assessment of subjective emotional valence and long-lasting impact of life events: development and psychometrics of the Stralsund Life Event List (SEL). BMC Psychiatry. 2018;18:105 https://doi.org/10.1186/s12888-018-1649-3

2. Binder EB. The role of FKBP5, a co-chaperone of the glucocorticoid receptor in the pathogenesis and therapy of affective and anxiety disorders. Psychoneuroendocrinology . 2009;34:S186-95. https://doi.org/10.1016/j.psyneuen.2009.05.021

3. Vermeer H, Hendriks-Stegeman BI, Van der Burg B, van Buul-Offers SC, Jansen M. Glucocorticoid-induced increase in lymphocytic FKBP51 messenger ribonucleic acid expression: a potential marker for glucocorticoid sensitivity, potency, and bioavailability. J Clin Endocrinol Metab. 2003;88:277-84. https://doi.org/10.1210/ jc.2002-020354

4. Wochnik GM, Ruegg J, Abel GA, Schmidt U, Holsboer F, Rein T. FK506-binding proteins 51 and 52 differentially regulate dynein interaction and nuclear translocation of the glucocorticoid receptor in mammalian cells. J Biol Chem. 2005;280:4609-16. https://doi.org/10.1074/jbc.M407498200

5. Klengel T, Mehta D, Anacker C, Rex-Haffner M, Pruessner JC, Pariante CM, et al. Allele-specific FKBP5 DNA demethylation mediates gene-childhood trauma interactions. Nat Neurosci. 2013;16:33-41. https://doi.org/10.1038/nn.3275

6. Binder EB, Salyakina D, Lichtner P, Wochnik GM, Ising M, Putz B, et al. Polymorphisms in FKBP5 are associated with increased recurrence of depressive episodes and rapid response to antidepressant treatment. Nat Genet. 2004;36:1319-25. https://doi.org/10.1038/ng1479

7. Binder EB, Bradley RG, Liu W, Epstein MP, Deveau TC, Mercer KB, et al. Association of FKBP5 polymorphisms and childhood abuse with risk of posttraumatic stress 
disorder symptoms in adults. JAMA. 2008;299:1291-305. https://doi.org/10.1001/ jama.299.11.1291

8. Denny WB, Valentine DL, Reynolds PD, Smith DF, Scammell JG. Squirrel monkey immunophilin FKBP51 is a potent inhibitor of glucocorticoid receptor binding. Endocrinology. 2000;141:4107-13. https://doi.org/10.1210/endo.141.11.7785

9. Höhne N, Poidinger M, Merz F, Pfister H, Bruckl T, Zimmermann P, et al. FKBP5 genotype-dependent DNA methylation and mRNA regulation after psychosocial stress in remitted depression and healthy controls. Int J Neuropsychopharmacol. 2014. https://doi.org/10.1093/ijnp/pyu087

10. Yehuda R, Daskalakis NP, Bierer LM, Bader HN, Klengel T, Holsboer F, Binder EB. Holocaust exposure induced intergenerational effects on FKBP5 methylation. Biol Psychiatry. 2016;80:372-80. https://doi.org/10.1016/j.biopsych.2015.08.005

11. Appel K, Schwahn C, Mahler J, Schulz A, Spitzer C, Fenske K, et al. Moderation of adult depression by a polymorphism in the FKBP5 gene and childhood physical abuse in the general population. Neuropsychopharmacology. 2011;36:1982-91. https://doi.org/10.1038/npp.2011.81

12. Zimmermann $P$, Bruckl T, Nocon A, Pfister $H$, Binder EB, Uhr $M$, et al. Interaction of FKBP5 gene variants and adverse life events in predicting depression onset: results from a 10-year prospective community study. Am J Psychiatry. 2011;168:1107-16. https://doi.org/10.1176/appi.ajp.2011.10111577

13. Handwerger K. Differential patterns of HPA activity and reactivity in adult posttraumatic stress disorder and major depressive disorder. Harv Rev Psychiatry. 2009;17:184-205. https://doi.org/10.1080/10673220902996775

14. Grabe HJ, Wittfeld K, Van der Auwera S, Janowitz D, Hegenscheid K, Habes M, et al. Effect of the interaction between childhood abuse and rs1360780 of the FKBP5 gene on gray matter volume in a general population sample. Hum Brain Mapp. 2016;37:1602-13. https://doi.org/10.1002/hbm.23123

15. Resmini E, Santos A, Aulinas A, Webb SM, Vives-Gilabert Y, Cox O, et al. Reduced DNA methylation of FKBP5 in Cushing's syndrome. Endocrine. 2016. https://doi. org/10.1007/s12020-016-1083-6

16. Han K-M, Won E, Sim Y, Kang J, Han C, Kim Y-K, et al. Influence of FKBP5 polymorphism and DNA methylation on structural changes of the brain in major depressive disorder. Sci Rep. 2017;7:42621 https://doi.org/10.1038/srep42621

17. Pogribny IP, Beland FA. DNA hypomethylation in the origin and pathogenesis of human diseases. Cell Mol Life Sci. 2009;66:2249-61. https://doi.org/10.1007/ s00018-009-0015-5

18. Goll MG, Bestor TH. Eukaryotic cytosine methyltransferases. Annu Rev Biochem. 2005;74:481-14. https://doi.org/10.1146/annurev.biochem.74.010904.153721

19. Li E, Zhang Y. DNA methylation in mammals. Cold Spring Harb Perspect Biol. 2014;6:a019133 https://doi.org/10.1101/cshperspect.a019133

20. Razin A, Riggs AD. DNA methylation and gene function. Science. 1980;210:604-10.

21. Piyasena C, Cartier J, Provençal N, Wiechmann T, Khulan B, Sunderasan R, et al. Dynamic changes in DNA methylation occur during the first year of life in preterm infants. Front Endocrinol (Lausanne). 2016;7:158 https://doi.org/10.3389/ fendo.2016.00158

22. Winkler BK, Lehnert $\mathrm{H}$, Oster $\mathrm{H}$, Kirchner $\mathrm{H}$, Harbeck B. FKBP5 methylation as a possible marker for cortisol state and transient cortisol exposure in healthy human subjects. Epigenomics. 2017;9:1279-86. https://doi.org/10.2217/epi-20170057

23. Völzke H, Alte D, Schmidt CO, Radke D, Lorbeer R, Friedrich N, et al. Cohort profile: the study of health in Pomerania. Int J Epidemiol. 2011;40:294-307. https://doi. org/10.1093/ije/dyp394

24. Ehrich M, Nelson MR, Stanssens P, Zabeau M, Liloglou T, Xinarianos G, et al. Quantitative high-throughput analysis of DNA methylation patterns by basespecific cleavage and mass spectrometry. Proc Natl Acad Sci USA. 2005;102:15785-90. https://doi.org/10.1073/pnas.0507816102

25. Schurmann C, Heim K, Schillert A, Blankenberg S, Carstensen M, Dorr M, et al. Analyzing illumina gene expression microarray data from different tissues: methodological aspects of data analysis in the metaxpress consortium. PLoS ONE. 2012;7:e50938 https://doi.org/10.1371/journal.pone.0050938

26. Bernstein DP, Stein JA, Newcomb MD, Walker E, Pogge D, Ahluvalia T, et al. Development and validation of a brief screening version of the Childhood Trauma Questionnaire. Child Abus Negl. 2003;27:169-90. https://doi.org/10.1016/ S0145-2134(02)00541-0
27. Wittchen H-U. DIA-X-Interviews: Manual für Screening Verfahren und Interview; Interviewheft Längsschnittuntersuchung (DIA-X-Lifetime); Ergänzungsheft (DIAX-Lifetime); Interviewheft Querschnittsuntersuchung (DIA-X-12 Monate); Ergänzungsheft (DIA-X-12 Monate). Frankfurt: Swets \& Zeitlinger; 1997.

28. Wittchen H-U, Pfister H. Diagnostisches Expertensystem für psychische Störungen. Frankfurt: Swets \& Zeitlinger; 1997.

29. Beck AT, Steer RA, Brown G. Manual for the Beck Depression Inventory-II. San Antonio: The Psychological Corporation; 1996.

30. Wahl I, Lowe B, Bjorner JB, Fischer F, Langs G, Voderholzer U, et al. Standardization of depression measurement: a common metric was developed for 11 selfreport depression measures. J Clin Epidemiol. 2014;67:73-86. https://doi.org/ 10.1016/j.jclinepi.2013.04.019

31. Hertel J, König J, Homuth G, Van der Auwera S, Wittfeld K, Pietzner M, et al. Evidence for stress-like alterations in the HPA-Axis in women taking oral contraceptives. Sci Rep. 2017;7:14111 https://doi.org/10.1038/s41598-017-13927-7

32. Papke LE, Wooldridge JM. Econometric methods for fractional response variables with an application to $401(\mathrm{k})$ plan participation rates. J Appl Econ. 1996;11:619-32. https://doi.org/10.1002/(SICI)1099-1255(199611)11:6<619::AIDJAE418>3.0.CO;2-1

33. Roy B, Shelton RC, Dwivedi Y. DNA methylation and expression of stress related genes in PBMC of MDD patients with and without serious suicidal ideation. J Psychiatr Res. 2017;89:115-24. https://doi.org/10.1016/j.jpsychires.2017.02.005

34. Wahl S, Fenske N, Zeilinger S, Suhre K, Gieger C, Waldenberger $M$, et al. On the potential of models for location and scale for genome-wide DNA methylation data. BMC Bioinforma. 2014;15:232 https://doi.org/10.1186/14712105-15-232

35. Danese A, Tan MT. Childhood maltreatment and obesity: systematic review and meta-analysis. Mol Psychiatry. 2014;19:544-54. https://doi.org/10.1038/ mp.2013.54

36. Dannlowski U, Stuhrmann A, Beutelmann V, Zwanzger P, Lenzen T, Grotegerd D, et al. Limbic scars: long-term consequences of childhood maltreatment revealed by functional and structural magnetic resonance imaging. Biol Psychiatry. 2012;71:286-93. https://doi.org/10.1016/j.biopsych.2011.10.021

37. Downs WR, Harrison L. Childhood maltreatment and the risk of substance problems in later life. Health Soc Care Community. 1998;6:35-46.

38. Li M, D'Arcy C, Meng X. Maltreatment in childhood substantially increases the risk of adult depression and anxiety in prospective cohort studies: systematic review, meta-analysis, and proportional attributable fractions. Psychol Med. 2016;46:717-30. https://doi.org/10.1017/S0033291715002743

39. Molendijk ML, Hoek HW, Brewerton TD, Elzinga BM. Childhood maltreatment and eating disorder pathology: a systematic review and dose-response meta-analysis. Psychol Med. 2017:1-15. https://doi.org/10.1017/S0033291716003561.

40. Lee RS, Tamashiro KLK, Yang X, Purcell RH, Harvey A, Willour VL, et al. Chronic corticosterone exposure increases expression and decreases deoxyribonucleic acid methylation of Fkbp5 in mice. Endocrinology. 2010;151:4332-43. https://doi. org/10.1210/en.2010-0225

41. Bustamante AC, Aiello AE, Guffanti G, Galea S, Wildman DE, Uddin M. FKBP5 DNA methylation does not mediate the association between childhood maltreatment and depression symptom severity in the Detroit Neighborhood Health Study. J Psychiatr Res. 2018;96:39-48. https://doi.org/10.1016/j.jpsychires.2017.09.016

42. Matsuda A, Asada Y, Takakuwa K, Sugita J, Murakami A, Ebihara N. DNA Methylation Analysis of Human Trabecular Meshwork Cells During Dexamethasone Stimulation. Invest Ophthalmol Vis Sci. 2015:56:3801-9. https://doi. org/10.1167/iovs.14-16008

43. Needham BL, Smith JA, Zhao W, Wang X, Mukherjee B, Kardia SLR, et al. Life course socioeconomic status and DNA methylation in genes related to stress reactivity and inflammation: The multi-ethnic study of atherosclerosis. Epigenetics. 2015;10:958-69. https://doi.org/10.1080/15592294.2015.1085139

44. Paquette AG, Lester BM, Koestler DC, Lesseur C, Armstrong DA, Marsit CJ. Placental FKBP5 genetic and epigenetic variation is associated with infant neurobehavioral outcomes in the RICHS cohort. PLoS ONE. 2014;9:e104913 https://doi. org/10.1371/journal.pone.0104913

45. Ewald ER, Wand GS, Seifuddin F, Yang X, Tamashiro KL, Potash JB, et al. Alterations in DNA methylation of Fkbp5 as a determinant of blood-brain correlation of glucocorticoid exposure. Psychoneuroendocrinology . 2014;44:112-22. https:// doi.org/10.1016/j.psyneuen.2014.03.003 